INTERNATIONAL DESIGN CONFERENCE - DESIGN 2018

https://doi.org/10.21278/idc.2018.0130

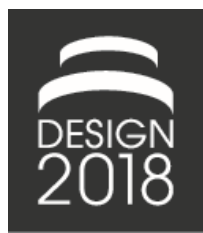

\title{
APPROXIMATION OF THE USER BEHAVIOUR IN A FULLY AUTOMATED VEHICLE REFERRING TO A STATIONARY PROTOTYPE-BASED RESEARCH STUDY
}

\author{
F. Fitzen, M. Amereller and K. Paetzold
}

\begin{abstract}
The technological progress of automated driving fundamentally affects the starting situation for developing the automotive interior. As the driver will not be occupied with driving tasks, a self-driving car should enable him and all passengers to productively shape their driving time in a new way. Basing on previous approaches of deriving interior functional requirements a literature review as well as a research study in a stationary prototype regarding relevant secondary activities have been conducted. The outcome is exemplified, embedded in a methodical approach and next steps are deduced.
\end{abstract}

Keywords: human centred design, user experience, requirements management, interior of the future, automated driving

\section{Introduction}

Capturing individual user needs is one of the fundamental challenges in the automotive development process. Especially the fluent transition to automated driving (Institute for Mobility Research, 2016) generates a whole new starting situation for the functional design of the vehicle interior, which is directly perceived by the user in particular. Hence the self-driving car allows the driver, as he is not occupied with primary driving tasks any more, and all other occupants to shape their time in the vehicle in a freer, more individual way (Rupp and King, 2010), which then can be used for non-driving related, so called secondary tasks (Parliament of Victoria, 2006) such as sleeping, eating and working (Fraunhofer IAO and Horváth \& Partners, 2016). The term 'secondary task' is often also described as 'secondary activity' or 'driver distraction' (Huemer and Vollrath, 2012). For designing the vehicle interior of an automated vehicle the prospective user behaviour is of particular importance. According to Tomforde (2007) there is a frequent lack of sufficient and precise information about presumable users and a comprehensive appreciation of users' needs for the purpose of taking this into account for decision making and strategy. In accordance with Tomforde (2007) this situation requires automobile manufacturers to already be aware of the future trends several years before the market launch of a new derivative in order to create prerequisites for the implementation in the early phase.

In a similar context Herbst (2017) was already concerned with deriving room functional customer requirements in the automotive development. The acquired methodology uses a systematic description of the function itself as a basis and recommends the situational selection out of three methodical modules: the interaction of the user with the product, a functional benchmarking as well as a customer satisfaction evaluation. The so ascertained customer requirements are unified using empirical assessment criteria and subsequently aggregated to an individual, customer related, room functional target image for every derivative. The modes of action and functioning are proven for the application 
on current development projects. When transferring this approach to the interior development of automated vehicles it occurs that there are only few competitors offering conditional automation vehicles, defined as level 3 vehicles (SAE International, 2014). High and full automation vehicles will not be available on the market at least until 2029, as VDA (2017) predicts. Thus no functional data regarding competitors as well as no customer satisfaction evaluation are available for automated vehicles at this point. The overall goal therefore needs to be to forecast the user behaviour in an automated vehicle, especially regarding secondary activities, although both future users and developers are currently not aware of exact predictions. Dealing with these challenges the users' interaction with the product itself, as in research studies in prototypes or interactive inquiries, can be a useful approach (DIN, 2010). Especially through the transition to automated driving it seems necessary to take secondary activities, which can be even more various than the activities performed in a vehicle nowadays, more into account. Several experts such as Claus Ehlers, head of 'society, vehicle concepts and human machine interaction' at Daimler, predict the vehicle of the future to be more of a living space as it is recognized today (Abele, 2010). The following chapter describes the state of the art regarding secondary activities in automated vehicles and therefore provides a basis for the user-centred approach acquired in Chapter 3. Built upon this methodology a research study in a stationary vehicle aiming at secondary activities was performed, whose results will then be explained and discussed.

\section{State of the art}

In order to fulfil the users' expectations despite the fact that the future user's behaviour is unknown it seems necessary to even more focus the user and his experiences and needs for the development. The identification of relevant secondary activities, which hereafter are also denoted as use cases (Institute for Mobility Research, 2016), is thereby essential. The following research questions are postulated:

1. What kind of methodology is able to involve the users' needs even more into the development of the interior of automated driving vehicles?

2. How will it be possible to integrate the changing user behaviour in automated driving vehicles into the functional design of the interior of the future?

\subsection{Research methodology}

Within the scope of a widespread literature search the state of the art was gathered and analysed by clustering the findings into five search fields. In the 'automated driving' field the current state of development in advanced driver assistance systems (ADAS) and the different levels of automation according to (SAE International, 2014) could be identified. Latter will have significant impact on the realisation of secondary activities as the required user attendance decreases with an increasing level of automation. Also the people's general attitude to automated driving as well as potentially occurring barriers between user and technology were taken into account. The field 'customer focus' targeted any existing studies regarding secondary activities in self-driving vehicles, also including official studies of other departments as well as studies concerning passenger activities in current vehicles. All in all 17 research studies were found to be investigated closer. The summarised findings acquired through a metaanalysis will be shown in the following Section 2.2. Another field could be identified with 'transportation'. This includes rail and air traffic, where passengers are confronted with similar spatial and time-wise circumstances and are also not obtained with driving tasks. The 'ergonomics' field intended to look for synergies for space within reach of the occupants which could be relevant for functionally designing specific concepts. Finally, the field 'interior of the future' focused on interior concepts that already have been presented by automotive manufacturers or suppliers on exhibitions. Insights that have been identified by Herbst (2017) also have influence in this field. Following up to the literature search a study in a stationary vehicle in a prototype regarding secondary activities was performed, whose findings will be described in Chapter 3. The results were then compared to the literature following in Chapter 4.

\subsection{Meta-analysis of existing research studies}

The search fields mentioned above helped in identifying 17 research studies which are concerned with secondary activities in automated vehicles or other means of transportation. Within the scope of these 
studies the opinions of over 50,000 study participants were captured, whereby the two biggest studies were already covering just under 40,000 persons (Lyons et al., 2007; Goodyear, 2016). All use cases mentioned in one of the studies were then clustered into the six categories of human needs identified by (Fraunhofer IAO and Horváth \& Partners, 2016). These categories are represented by 'communication', 'productivity', 'basic needs' and 'well-being' as well as 'information' and 'entertainment'. Every category contains several so called 'service groups', of which there are 21 in total. 'Eating/drinking' and 'sleeping' are therefore service groups of 'basic needs'. All in all 63 secondary activities from all research studies have been assigned to the service groups in order to structure the found literature first. Table 1 shows a categorisation using the example of 'basic needs'.

Table 1. Exemplary categorisation of the user need 'basic needs' according to Fraunhofer IAO and Horváth \& Partners (2016)

\begin{tabular}{|c|c|c|}
\hline User need & Service group & Secondary activity/use case \\
\hline \multirow{12}{*}{ Basic needs } & \multirow{5}{*}{ Eating/drinking } & Eating \\
\hline & & Preparing food \\
\hline & & Drinking \\
\hline & & Tempering items \\
\hline & & Making coffee \\
\hline & \multirow{2}{*}{ Clothing } & Tying one's tie \\
\hline & & Changing clothes \\
\hline & \multirow{2}{*}{ Sleeping } & Relaxing \\
\hline & & Sleeping deeply \\
\hline & \multirow{3}{*}{ Washing/cleaning } & Polishing shoes \\
\hline & & Ironing \\
\hline & & Drying clothes in the trunk \\
\hline
\end{tabular}

After structuring the existing data a meta-analysis was performed. The goal of this meta-analysis was to derive a list of priority for secondary activities in automated vehicles resulting from the state of the art studies and inquiries. In order to guarantee a scientific standard all studies were assessed with established quality criteria of quantitative research meaning reliability, validity and objectivity (Schnapp et al., 2006). Every study was judged with fulfilled, restrictedly fulfilled and not fulfilled regarding every quality criterion. In case of a not fulfilled assessment, the study was not used for the meta-analysis as it did not match the requirements of the scientific standard. One example can be named by the sample size of a study, which was set to a minimum of $n=30$ study participants due to the recommendation of Bubb (2003), who identified this as a number for significant results in driving experiments. Finally a method for ensuring the accuracy of the results was applied with the $3 \sigma$-rule according to Töpfer (2003). This method identifies outliers within a certain range and eradicates them in an iterative process. Therefore in a first step, all measurements not laying within $\pm 3 \sigma$ around the average are eliminated. Afterwards, the new average is calculated and the $3 \sigma$-rule is applied again. The result is a revised data set without outliers and with all activities laying in the addressed range (Runkler, 2010).

Using this approach on all studies and use cases a list of priority on the basis of existing research regarding secondary activities can be generated. As not every use case was mentioned in every study, the number of the total study participants varies from use case to use case. Table 2 shows the top 10 secondary activities as the result of this meta-analysis. It stands out that drinking, enjoying the scenery and eating are all more conventional activities, whereas emailing $(30.0 \%)$ or other spare time activities such as watching a movie (19.6\%) or sleeping (15.9\%) seem to be less attractive to the study participants. Having a closer look at the existing research some of the considered studies did not include any quantitative results regarding the relevance of a use case in relation to their amount of study participants. If there was no specification made, the study could not be of any use for this meta-analysis. A few use cases also showed a high standard deviation which was understood as evidence of disagreement of the study participants. As one of the reasons the great inexperience of the user with the situation of driving in an automated vehicle could be identified (Schoettle and Sivak, 2014). This poses 
the question if inquiries of the user are an eligible source for identifying secondary activities as they not only are not used to the situation but as well, for most of the study participants, do not have sophisticated knowledge about the possibilities of automated vehicles either (Fraedrich et al., 2016).

Table 2. Top 10 use cases as a result of the meta-analysis

\begin{tabular}{|c|c|c|c|}
\hline Ranking & Use case & $\begin{array}{c}\text { Total number of study } \\
\text { participants }\end{array}$ & $\begin{array}{c}\text { Proportion of all study } \\
\text { participants [\%] }\end{array}$ \\
\hline 1 & Having videoconferences/virtual meetings & 1,533 & $69.6 \%$ \\
\hline 2 & Drinking & 333 & $59.5 \%$ \\
\hline 3 & Enjoying the scenery & 34,458 & $54.2 \%$ \\
\hline 4 & Preparing food & 1,800 & $51.8 \%$ \\
\hline 5 & Eating & 6,989 & $47.7 \%$ \\
\hline 6 & Reading & 48,225 & $43.1 \%$ \\
\hline 7 & Using social media & 2,050 & $41.2 \%$ \\
\hline 8 & Making a call & 19,469 & $40.5 \%$ \\
\hline 9 & Texting & 14,553 & $39.2 \%$ \\
\hline 10 & Surfing the internet & 13,601 & $37.2 \%$ \\
\hline
\end{tabular}

Therefore the experience of the users riding in an automated vehicle or at least creating a similar atmosphere around them can be a legit approach to validate the state of the art which was reinforced by experts inside the company. Furthermore Herrler (2006) stated that experiments under laboratory conditions as well as real driving scenarios should be taken into account when developing a vehicle. Another outcome of the meta-analysis can be mentioned by the fact that there was no differentiation made regarding target groups, target markets or automation levels. Referring to Herbst (2017), who developed specific target images for each derivative, the existing data base does not enable individual priorities for secondary activities in automated vehicles.

\subsection{Summary of the state of the art}

Summarizing the meta-analysis secondary activities have already been part of some scientific research. Data of around 50,000 study participants have been collected in different study designs and approaches on what they will be supposed to do in an automated vehicle. It stands out that the number of study participants that were able to select the respective use case varies heavily between 333 and 48,225. Also, it came up that most of the respondents feel quite familiar with performing conventional activities such as drinking, eating and enjoying the scenery. Use cases as videoconferences and preparing food also seem to be a relevant activity according to the existing research. Moving on in the priority list it is noticeable that already the tenth use case out of 63 only has just above a third of all respondents that can imagine this during a ride in an automated car, which can on the one hand be again related to the unawareness of people with the situation. On the other hand this can be evidence that people do not want to pursue most of the activities, which has to be clarified in further research. If it comes to specific customer-, vehicle-related data, which made a significant difference in designing the current vehicle interior (Herbst, 2017), the data base seems not sufficient. Also the possibilities and challenges of different automation levels coming with secondary activities lead to the conclusion that further research has to be initiated. In the context of advanced driver assistance systems Breuer et al. (2015) stated that in the end only the inexperienced future user can lead the developers to the actual requirements. In order to validate the existing research a real experiment on test persons simulating a situation similar to an automated vehicle seems indispensable. Therefore, first of all, a systematic approach for generating individual use case data and subsequently deriving functional requirements to the interior is necessary, which will be described in the following section.

\section{User-centred approach}

The ascertainable approach is based on Herbst (2017) where functional requirements were assessed with a customer satisfaction evaluation, a functional benchmarking as well as the interaction of the user with 
the product. For the first two methodological modules no data is available regarding the functional interior of automated vehicles, especially for secondary activities. Subsequently it is essential to focus on the interaction of the future user with the vehicle interior. The therefore needed approach aims to assess user data regarding secondary activities in an automated vehicle depending on certain parameters as target market, customer group and vehicle size and its targeted level of automation.

\subsection{Method}

For the purpose of covering qualitative and quantitative studies three methods were planned to assess the data. First of all, a standardised, quantitative questionnaire should be included to get a good overview over different user groups and to guarantee a high number of study participants. The so conducted standardised data collection will be essential for a profound database in order to show a differentiation of use case priorities depending on e.g. customer group, target vehicle. Secondly, a qualitative observation combined with a quantitative questionnaire is able to gather information on how test persons interact with their surroundings, which seems geared to the needs of this research. An observation report therefore ensures a completeness of recognized behaviour, the added 'thinking aloud' method supports the collection of user behaviour in a qualitative observation according to Sprenger (2008). It is particularly suitable for study setups in which changes during the study might occur (Sprenger, 2008). Finally, a mix of qualitative and quantitative observation as well as a quantitative questionnaire will provide numerical observation data of the test persons, ideally confirming the results gathered in previous inquiries. Main output of this method will be frequencies and durations of how long test persons perform which secondary activities in the vehicle (Döring and Bortz, 2016).

Existing literature of a development process also recommends varying the research study setup due to supposedly different focuses. A theoretical study first of all seems useful in terms of a validation of the state of the art. This should also lead to a more profound and specific data base with a significant number of study participants. In the context of the user interface a study in a stationary vehicle is often performed during the initial or concept phase in order to identify the main sources of non-conformances. As described above the supposable difference between a study in laboratory conditions, such as a stationary vehicle, and a study in real surroundings, such as a moving vehicle, should be taken into account (Herrler, 2006). Breuer et al. (2015), within the scope of advanced driver assistance systems, also remarks that experiments with experts can be helpful and necessary in the early concept phase as a preliminary stage to research studies with inexperienced users. This adds up to three different study setups: a theoretical study, a study in a stationary vehicle and a study in a moving vehicle. A theoretical study will be performed as a quantitative questionnaire, a study in a stationary vehicle should combine a quantitative questionnaire and a qualitative observation and will be conducted on experts. In the end, a study in a moving vehicle will involve a combination of all three methods using inquiries before and after the study as a comparison (Table 3). All other methods used in all of the study setups are held during the study itself. The research study in a stationary vehicle was already performed on a prototype, whereby its results are depicted in the following section.

Table 3. Assignment of data collection type to the used methods

\begin{tabular}{|c|c|c|c|}
\hline \multirow{2}{*}{ Research study setup } & \multicolumn{3}{|c|}{ Methods } \\
\cline { 2 - 4 } & $\begin{array}{c}\text { Quantitative } \\
\text { Questionnaire }\end{array}$ & $\begin{array}{c}\text { Qualitative } \\
\text { Observation }\end{array}$ & $\begin{array}{c}\text { Quantitative } \\
\text { Observation }\end{array}$ \\
\hline Theoretically & During & & \\
\hline In a stationary vehicle & During & During & During \\
\hline In a moving vehicle & Before/after & During & \\
\hline
\end{tabular}

\subsection{Stationary prototype-based research study}

The study was performed in a concept vehicle containing an innovative seat concept. The vehicle was located in a closed room, while the test persons were given a specific situation during automated driving without any driving tasks. Due to non-disclosure the exact concept of the vehicle cannot be detailed any 
further. The main goal was to investigate the users' behaviour in this seat concept simulating the described surroundings.

After letting the test person get comfortable with the surroundings of the concept for a few minutes, the study itself started using the guidelines, that were created and pre-tested according to Döring and Bortz (2016). The first part was to judge the seating comfort in two different seating positions right after each other in order to be able to compare the two positions. This was followed by general questions referring to the use of vehicles, e.g. what kind of car the test person uses at the moment or how often they use it. This all adds up to a classification of the test person into target groups. For the next step, the test persons were given a scenario in which the vehicle drives fully automated through a city or on a highway.

They would not have to pursue any driving tasks and could have their time spend with secondary activities according to their preferences. Therefore the test persons were told to give an estimation on which secondary activity is imaginable at all, crossing all relevant from a list of 23 use cases. This list was pre-selected from the state of the art according to given surroundings of the seat concept, the persons were also invited to add any activity that came to their mind. Subsequently a prioritisation of the individually most important activities from top 1 to top 5 was asked, as the test persons were then able to try out their top 5 use cases for around two minutes each with use case relevant items and describing their thoughts afterwards. The items were identified through literature review and selectively transferred to other use cases using expert knowledge in the interior department. The test persons were also able to make adjustments to their estimation that was made before, followed by an assessment of how often and how long they could imagine using the seat concept. A user information block concluded the study, which was also intended to categorize the test persons into target groups as explained above. All in all, 69 persons were invited of which 30 then participated in the study. The group was composed by 9 female and 21 male test persons coming from different departments such as development, production, purchasing and finance. Mainly younger people, meaning under 40, participated in the study (Table 4).

Table 4. Composition of the test persons regarding age groups

\begin{tabular}{|c|c|c|c|c|}
\hline Age group & $<\mathbf{3 0}$ & $\mathbf{3 0 - 3 9}$ & $\mathbf{4 0 - 4 9}$ & $>\mathbf{5 0}$ \\
\hline Number of test persons & 16 & 10 & 2 & 2 \\
\hline
\end{tabular}

\section{Results of the research study in a stationary vehicle}

The following section initially provides an overall description of the study results regarding use cases in an automated vehicle also giving a detailed parameter-based insight into the findings. Afterwards the results are compared to the assessment of the literature review.

For the purpose of ranking the use cases first of all the percentage of how many test persons can imagine performing a use case in the vehicle were contrasted. It stands out that talking to other passengers is the most relevant secondary activity in the eyes of the test persons. Looking at this simple yes or noquestion, $97 \%$ of all persons guess that they would need this use case (Figure 1). This is followed by relaxing, making a call, surfing the internet and reading which appear to be activities commonly pursued in their own living room. As the test persons were also able to prioritise their top 5 from the before selected ones, a linear weighting was introduced. The top activity was weighted with 6 points going linearly down to 2 points for the top 5 activity. Every other ticked activity was weighted with 1 point. This is also depicted in Figure 1 showing that relaxing is the most prioritised use case overall. This use case was applied to be the reference for the grading of all other use cases.

Thus the test persons rated talking to other passengers, surfing the internet and listening to music as the next important use cases. It occurs that surfing the internet and listening to music get more important with the linear weighting, whereas talking to other passengers and making a call lose importance. This means that basically a lot of test persons can imagine a use case, but when it comes to prioritising other use cases gain more in importance, which is shown by the light grey bars in Figure 1. All in all, the test persons were also invited to add specific secondary activities that they missed. Only one test person wanted to include stretching as a separate activity which could also be seen under the aspect fitness. Otherwise, all other test persons did not add any other tasks. 


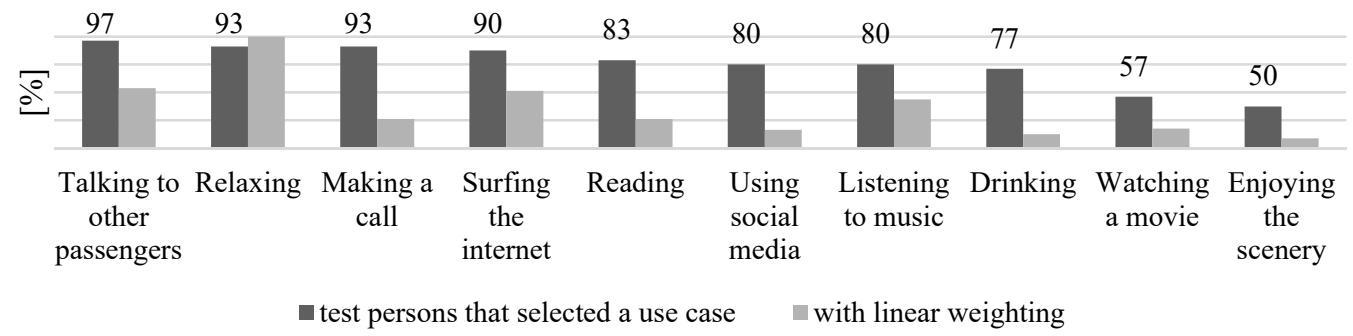

Figure 1. Prioritisation of the use cases

For statistical evaluation the Mann-Whitney-U-Test was performed in order to determine dependences in two independent samples. The significance level was set to $10 \%$, which can be used investigating novel phenomena according to Döring and Bortz (2016). The confidence level therefore adds up to $90 \%$. For sample sizes $n=30$ or larger, the asymptotic significance is the relevant measurement. The results of this test show, that there are significant dependences regarding age, gender and proportion of office work of the test persons, which concerns the following secondary activities: eating and drinking get more relevant with an increasing age group of the test persons, whereas drinking is also more relevant for test persons with a higher proportion of office work. Talking to other passengers, painting, playing games on the smartphone and brain callisthenics is more essential for younger people. On the other hand, brain callisthenics was also more important to male test persons compared to females. Writing emails also seemed most attracting to the age group 30-39. As a sum up, these results as a function of age group, gender and proportion of office work show a first impression that the choosing of relevant secondary activities is dependent on various parameters.

To identify further relationships between secondary activities a principle component analysis was performed. Therefore, the matrix with all secondary activities was revised until the Kaiser-Meyer-Olkin Measure of Sampling Adequacy was higher than .6 $(\mathrm{KMO}=.645)$, as recommended for a useful data set (Backhaus, 2011). By means of the scree plot and the elbow method, the number of components was found to be three. The resulting rotated component matrix was filtered according to Backhaus (2011) in order to only have highly relevant values larger than .5 (see Figure 2). It shows that the secondary activities working, brain callisthenics, eating, playing board games, painting and writing emails refer to component 1 and therefore promote each other. A test person that chose working as relevant preferably wanted to perform other creative, claiming and productive activities as well. Expectedly, working and writing emails belong to the same component which can therefore be interpreted as a combined secondary activity for office tasks. Analogously, surfing the internet, reading the news and making a call, which can be allocated to component 2 , are mostly smartphone related secondary activities and are preferred by test persons altogether. It also can be concluded that reading the news is preferably done on a smartphone, as component 2 also contains the smartphone activity surfing the internet. The third component seems to be quite diverse, as texting has positive influence and enjoying the scenery as well as talking to other passengers have negative impact on it. This can be interpreted the way that test persons who preferably do texting as a secondary activity, do not want to enjoy the scenery and talk to other passengers. Whereas texting is a more isolated task, the other two are more interactive and integrate the test persons more in their surroundings. All in all, the three components show the cohesiveness of some secondary activities which can also be used for characterising these activities in the further research.

Through the study setup it is also possible to identify the use cases that were prioritised higher or lower after the test persons tried them out. It occurs that surfing the internet was increasingly perceived as pleasant. This applies to five out of 15 test persons which chose this use case in their top 5. On the other hand, texting ( 2 out of 4 ) and reading ( 3 out of 9) were estimated less relevant after the try-out session. All in all, only less than $5 \%$ of all use cases were completely discarded by the test persons which generally indicates interest in spending the time in an automated vehicle in a more productive way. 


\begin{tabular}{|l|c|c|c|}
\hline \multirow{2}{*}{} & \multicolumn{3}{|c|}{ Component } \\
\cline { 2 - 4 } & 1 & 2 & 3 \\
\hline Working &, 777 & & \\
Brain callisthenics &, 746 & & \\
Eating &, 637 & & \\
Playing board games &, 634 & & \\
Painting &, 603 & & \\
Writing emails &, 602 &, 759 & \\
Surfing the internet & &, 723 & \\
Reading the news & &, 703 & \\
Making a call & & & \\
Drinking & & &,- 826 \\
Reading & & &,- 502 \\
Business Meeting \\
Enjoying the scenery
\end{tabular}

Figure 2. Rotated component matrix of secondary activities

\section{Discussion}

First of all, when comparing the meta-analysis and the research study, it stands out that six out of ten use cases are in the top 10 of both results. This can be assessed with Figure 3 which depicts the rankings of all top 10 use cases of literature search and research study. Especially medium rated use cases such as making a call, reading or using social media were estimated similarly by test persons of the study as well as study participants of the literature review.

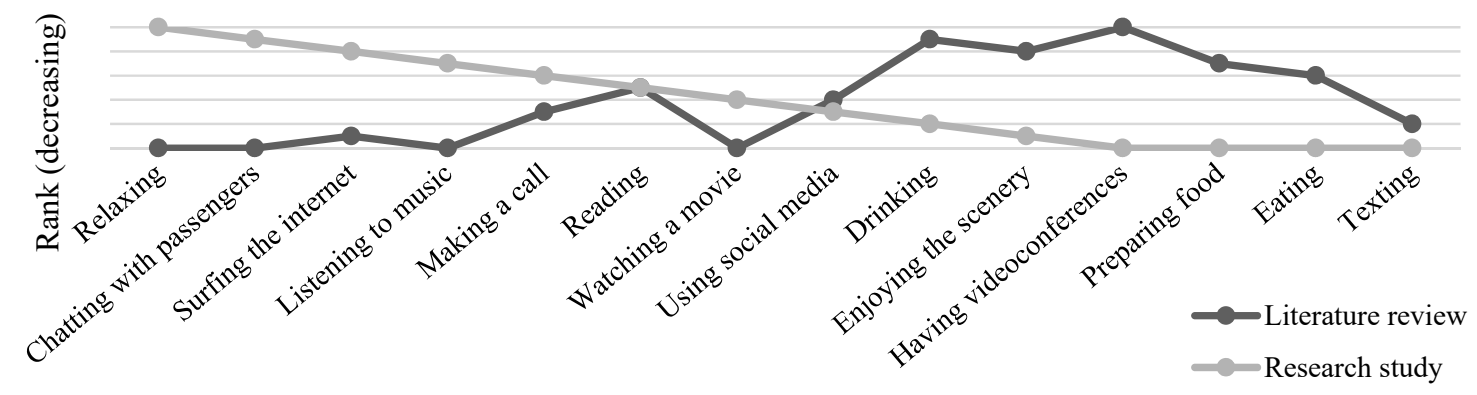

Figure 3. Top use cases of study results compared to the literature review

On the other hand, the most important rated activities relaxing, talking to other passengers and listening to music in the study are not part of the top 10 in literature. The same can be stated for videoconferences and preparing food the other way round. One theory therefore is that the personal experience of a user, even in a stationary vehicle, has influence on the imagination of use cases. This can be expressed in the way that they can imagine new use cases at all, or they feel unconsciously restricted in their possibilities. Another reason can be the different inquiry setups with which the user data was assessed for the literature review. Prospective users were asked in person, via telephone or with the help of a questionnaire, on the street, in other modes of transportation during the trip or at sitting on their desk at work and at home, which also might have had impact on their use case estimations. All in all, an overlap of the findings in literature review and the performed research study can be determined. Some of the use cases were prioritised with similar importance by both groups. At the same time, a few use cases can only be found 
in the top results of one assessment method, wherefore some reasons were named before. The findings of the study therefore have to be confirmed and extended in further research.

\section{Conclusion}

A general dependency of relevant use cases depending on the parameters already used in the study has been identified. This is intended to be confirmed with a higher number of study participants. On the other hand, there was a recognizable difference between results of the study and the state of the art. This can also be partly confirmed by Herrler (2006), who stated a significant difference in results of theory and real experiments. To bring the two aspects together a large standardised inquiry with at least 100 study participants is intended to check the dependency on parameters as well as a potential difference to the performed study regarding prioritised use cases. As a follow up, a research study investigating the users' behaviour in a stationary vehicle with a dynamic driving scene simulation as well as a real experiment with a prototype on public streets as a test ground is intended in order to assess the behaviour of future users in nearly optimum real surroundings. As a study setup a combination of an interview before and after the test drive as performed in the stationary study as well as the method of thinking aloud seems to be a valid way to assess potential real behaviour according to Sprenger (2008). All results meaning the state of the art, the stationary study, the theoretical inquiry as well as the driving experiment are to be aggregated and validated in the end. The aim of further research is to develop a multivariate tool that generates an individual list of relevant use cases depending on parameters such as vehicle class, derivative, target group, target market, gender, vehicle use and planned automation level. The logic behind this calculation through multivariate linking must therefore be worked out through the mentioned additional studies and inquiries in the further research process and systematically modelled, for example on the basis of exclusion criteria or decision trees. Basing on these vehicle-specific use cases, a method for deriving functional requirements and estimating the extent of changes required in comparison to current vehicles is to be developed on the basis of functional and geometrical correlations as well as expert assessments. For those functional requirements that cannot be implemented in an existing component guidance will have to be derived. This tool-supported approach is intended to lay the foundation for a systematic component development of the interior of the future based on use cases. In this way, an important contribution is to be made to the transition to automated driving and the vision of spare time in the vehicle.

\section{References}

Abele, R. (2010), Die Komforterfahrung. [online] Daimler Technicity. Available at: https://issuu.com/daimlertechnicity/docs/technicity_d_lowres (accessed 10.10.2017).

Backhaus, K. (2011), Multivariate Analysemethoden: Eine anwendungsorientierte Einführung, Springer, Berlin, Germany. https://doi.org/10.1007/978-3-642-16491-0

Bortz, J. and Döring, N. (2005), Forschungsmethoden und Evaluation für Human und Sozialwissenschaftler, Springer Medizin, Heidelberg.

Breuer, J., von Hugo, C., Mücke, S. and Tattersall, S. (2015), "Nutzerorientierte Bewertungsverfahren von Fahrerassistenzsystemen”, In: Winner, H., Hakuli, S., Lotz, F. and Singer, C. (Eds.), Handbuch Fahrerassistenzsysteme: Grundlagen, Komponenten und Systeme für aktive Sicherheit und Komfort, Springer Fachmedien Wiesbaden, pp. 183-196. https://doi.org/10.1007/978-3-658-05734-3_12

Bubb, H. (2003), "Wie viele Probanden braucht man für allgemeine Erkenntnisse aus Fahrversuchen?", Proceedings of Darmstädter Kolloquium Mensch \& Fahrzeug, Technical University Darmstadt, Germany, April 03-04, 2003, VDI-Verlag, Düsseldorf, pp. 26-39.

DIN (2010), DIN EN ISO 9241-210 - Ergonomics of human-system interaction, DIN Deutsches Institut für Normung e.V.

Döring, N. and Bortz, J. (2016), "Datenerhebung”, In: Döring, N. and Bortz, J. (Eds.), Forschungsmethoden und Evaluation, Springer-Verlag, Berlin and Heidelberg, pp. 321-578. https://doi.org/10.1007/978-3-642-410895_10

Fraedrich, E., Cyganski, R., Wolf, I. and Lenz, B. (2016), User Perspectives on Autonomous Driving: A Use-CaseDriven Study in Germany, Humboldt University, Berlin, Germany.

Fraunhofer IAO and Horváth \& Partners (2016), The Value of Time: Nutzerbezogene Service-Potenziale durch autonomes Fahren, Fraunhofer IAO and Horváth \& Partners, Stuttgart, Germany. 
Goodyear (2016), Autonomous Vehicles: Negotiating a place on the road, Think Good Mobility, Goodyear, London, UK.

Herbst, L.-M. (2017), Entwicklung einer Methodik zur Ableitung raumfunktionaler Kundenanforderungen in der Automobilentwicklung, PhD thesis, Technical University of Munich, Munich, Germany.

Herrler, M. (2006), Systemergonomie und Usability-Tests bei bildschirmbasierten Bedienoberflächen im Automobil, $\mathrm{PhD}$ thesis, Technical University of Munich, Munich, Germany.

Huemer, A.K. and Vollrath, M. (2012), Ablenkung durch fahrfremde Tätigkeiten - Machbarkeitsstudie, Bundesanstalt für Straßenwesen, Bremerhaven, Germany.

Institute for Mobility Research (2016), Autonomous Driving: The Impact of Vehicle Automation on Mobility Behaviour, Institute for Mobility Research, Berlin, Germany.

Lyons, G., Jain, J. and Holley, D. (2007), "The use of travel time by rail passengers in Great Britain", Transportation Research Part A: Policy and Practice, Vol. 41 No. 1, pp. 107-120. https://doi.org/10.1016/j.tra.2006.05.012

Parliament of Victoria (2006), Report of the Parliament of Victoria Road Safety Committee on the Inquiry into Driver Distraction, Parliament of Victoria, Melbourne, Australia.

Runkler, T.A. (2010), Data Mining: Methoden und Algorithmen intelligenter Datenanalyse, Springer, Berlin, Germany. https://doi.org/10.1007/978-3-8348-9353-6

Rupp, J.D. and King, A.G. (2010), Autonomous Driving - A Practical Roadmap, SAE International and Ford Motor Company, Dearborn, USA. https://doi.org/10.4271/2010-01-2335

SAE International (2014), Automated Driving: Levels of Driving Automation are defined in new SAE International Standard J3016, SAE International, Warrendale and Troy, USA.

Schnapp, K.-U., Schindler, D., Gschwend, T. and Behnke, J. (2006), "Qualitative und quantitative Zugänge. Eine integrative Perspektive", In: Behnke, J. (Ed.), Methoden der Politikwissenschaft: Neuere qualitative und quantitative Analyseverfahren, Nomos, Baden-Baden, Germany, pp. 11-26.

Schoettle, B. and Sivak, M. (2014), A Survey of Public Opinion about Autonomous and Self-Driving Vehicles in the U.S., the U.K., and Australia, University of Michigan, Transportation Research Institute, Michigan, USA. https://doi.org/10.1016/j.trf.2015.04.014

Sprenger, R. (2008), Empirische Forschungsmethoden für die Evaluation visueller Fahrerassistenzsysteme im Kraftfahrzeug, PhD thesis, Institut für Humanwissenschaften, University Paderborn, Paderborn, Germany.

Tomforde, J. (2007), "Entwicklung und Design von Freizeitmobilen”, In: Braess, H.-H. and Seiffert, U. (Eds.), Automobildesign und Technik: Formgebung, Funktionalität, Technik, Friedr. Vieweg \& Sohn Verlag, GWV Fachverlage GmbH Wiesbaden, Wiesbaden, Germany, pp. 202-216. https://doi.org/10.1007/978-3-83489411-3 13

Töpfer, A. (2003), Six Sigma: Konzeption und Erfolgsbeispiele, Springer-Verlag, Berlin and Heidelberg, Germany. https://doi.org/10.1007/978-3-662-09916-2

VDA (2017), Automatisiertes Fahren. [online] VDA. Available at: https://www.vda.de/de/themen/innovationund-technik/automatisiertes-fahren/automatisiertes-fahren.html (accessed 17.08.2017).

Florian Fitzen, PhD Candidate

BMW Group, Interior

Knorrstraße 147, 80788 München, Germany

Email: florian.fitzen@bmw.de 